\title{
Application of Multidisciplinary Team (MDT) in The Treatment of Severe Maxillofacial Trauma: A Retrospective Study
}

\section{Xu Sun}

The First Hospital of China Medical University

\section{Shuang Tong}

The First Hospital of China Medical University

\section{Shude Yang}

The First Hospital of China Medical University

Shu Guo ( $D$ sguo@cmu.edu.cn )

The First Hospital of China Medical University

\section{Research Article}

Keywords: Maxillofacial trauma, Jaw fracture, Multidisciplinary team(MDT)

Posted Date: April 13th, 2021

DOI: https://doi.org/10.21203/rs.3.rs-400538/v1

License: (c) (i) This work is licensed under a Creative Commons Attribution 4.0 International License. Read Full License 
Application of multidisciplinary team (MDT) in the treatment of severe maxillofacial trauma: a retrospective study

Xu Sun, Shuang Tong, Shude Yang, Shu Guo

Correspondence: Shu Guo, sguo@cmu.edu.cn

Department of Plastic Surgery, The First Hospital of China Medical University,

No.155, Nanjing North Street, Shenyang 110001, China

Full list of author information is available at the end of the article.

\begin{abstract}
Background: The severe maxillofacial injuries often involves multiple systems and locations, requiring multiple surgical specialties to participate in injury assessment, emergency surgery and follow-up planned staged surgery, thus requiring the participation and guidance of a multidisciplinary team. This study explores the treatment effect of this model on patients with severe maxillofacial trauma, so as to better promote the standard of severe maxillofacial trauma treatment, aiming to establish a scientific and standardized system of severe trauma treatment.
\end{abstract}

Method: We retrospectively analyzed the treatment process of 173 patients with severe maxillofacial trauma, including timely, orderly, and efficient out-of-hospital emergency treatment. Maxillofacial surgery led the in-hospital treatment, and coordinated with other disciplines to relieve respiratory obstruction, control bleeding, correct shock and save lives. We summarized the experience in the treatment of jaw fracture reduction and internal fixation after the condition was stable.

Result : With the specialist treatment and close multidisciplinary cooperation, the treatment effect was good, and the reduction and internal fixation of jaw fractures had achieved satisfactory results. Conclusion: Multidisciplinary coordinated emergency treatment of severe maxillofacial trauma is an effective measure to improve the success rate of treatment.

Keywords: Maxillofacial trauma, Jaw fracture, Multidisciplinary team(MDT)

\title{
Background
}


Craniomaxillofacial is one of the most vulnerable parts of the human body. Severe maxillofacial trauma is often accompanied by multiple injuries throughout the body, especially craniocerebral injury, multiple fractures of the limbs and pelvis, and facial nerve injury[1]. As the first-visit maxillofacial surgeon, he/she must adhere to the holistic concept during the emergency treatment process. It not only requires timely specialist treatment, but also multidisciplinary collaboration and effective systematic diagnosis and treatment measures, to achieve a more satisfactory effect.

From January 2018 to December 2020, the plastic surgery department of the First Hospital of China Medical University admitted a total of 268 maxillofacial trauma patients, including 173 patients who were involved in multidisciplinary joint diagnosis and treatment. A retrospective analysis of these patients is now reported as follows.

\section{Methods}

\section{General information}

Among the 173 cases in this group, there were 111 males and 62 females. The ratio of male to female was about $2: 1$; the age range was 12 to 87 years, with an average age of 35.6 years. Causes of injury: 121 cases (69.9\%) for traffic accidents, 32 cases (18.5\%) for fall injuries, and 20 cases (11.5\%) for other injuries. All had different degrees of soft tissue injury, including 71 cases with open wounds, accounting for $41.0 \%$.

This study has been approved by the Ethics Committee of The First Hospital of China Medical University. Informed consent is waived for this retrospective study by the Ethics Committee of The First Hospital of China Medical University. We confirmed all methods were carried out in accordance with relevant guidelines and regulations.

Injury assessment Examinations of mandibular 3D-CT, brain CT, and fracture sites of limbs were performed for patients who were considered for suspected fracture diagnosis. Results: There were 94 cases of zygomatic-maxillary complex fractures, 34 cases of nasoorbital ethmoidal fractures, 45 cases of mandibular fractures, and 102 cases of multiple types of composite fractures. CT scan of the brain showed 87 cases of frontotemporal lobe contusion, including 19 cases of intracerebral hematoma, 27 cases of subdural hematoma, and 13 cases of epidural hematoma. This group had 34 cases of combined limb and pelvic fractures, 3 cases of cervical spine fractures and cervical 
spinal cord injuries, and 28 cases of cerebrospinal fluid leakage.

\section{Treatment}

Patients with severe cranio-maxillofacial injuries need to be given necessary out-ofhospital emergency treatment, such as rapid removal of oral and nasal secretions and foreign bodies to keep the respiratory tract unobstructed; establishment of emergency venous channels, timely infusion of balancing fluid, blood volume supplementation, correction of hemorrhagic shock; rapid identification of active bleeding points on maxillofacial wounds, clamp or pack compression to stop bleeding[2]. After admitted to our hospital, information-based multidisciplinary collaborative treatment was carried out. The multidisciplinary team (MDT) was mainly composed of experts in the emergency department, trauma center, plastic surgery, neurosurgery, orthopedics, and ophthalmology. For patients with oral bleeding, respiratory obstruction and suffocation, the anesthesiologist was asked to perform emergency tracheal intubation to relieve the suffocation, debride, stop bleeding, and suture; if there was a hematoma in the neck or floor of the mouth, an emergency tracheotomy was performed; for the patients with spine fractures, paid attention to avoid causing or aggravating cervical spinal cord injury during the inspection and handling; patients with rib fractures and blood pneumothorax should be consulted by a thoracic surgeon for closed thoracic drainage, and rib fractures should be reset and fixed; patients with severe craniocerebral injuries should be consulted by a neurosurgeon, referring to the surgical treatment guidelines for craniocerebral trauma, to prioritize the treatment of craniocerebral injury, remove the brain contusion and/or hematoma with obvious space-occupying effect by craniotomy, and perform the decompressive craniectomy for patients with severe brain swelling during the operation; dehydration, reduction of intracranial pressure, and antiinfection treatment were given after surgery; for maxillofacial bone fractures with bone defects, displacement or abnormal mobility of the fractured segment, rigid internal fixation, intermaxillary ligation, and soft tissue repair were performed as soon as possible after the craniocerebral trauma was stabilized, to restore various physiological functions and appearance. Patients with orbital floor fractures and ocular trauma were treated with jaw fracture reduction and fixation as well as orbital floor repair; patients 
with limb fractures and pelvic fractures were transferred to orthopedic department, and jaw fracture reduction and internal fixation were performed at the same time or at the second stage of orthopedic surgical treatment.

Results 173 patients were treated by plastic surgery specialists, and under the multidisciplinary coordination with other related departments, especially emergency department, critical care medicine, anesthesiology, ophthalmology, neurosurgery, and orthopedics, the rescue was successful, and the reduction and internal fixation of jaw fractures achieved satisfactory results.

\section{Typical cases}

Case 1 A 32-year-old male patient suffered multiple maxillofacial bone fractures, brain contusions, rib fractures, lung contusions, and palate lacerations due to traffic accidents. He was given facial debridement, suture and tracheotomy in the primary hospital. When he arrived at the emergency department of our hospital, the patient was unconscious. After consulted by neurosurgery, thoracic surgery, trauma center and other departments, with a GCS score of 5T, he received closed thoracic drainage, and was admitted to ICU for treatment. After active treatment, the patient's condition gradually stabilized, his consciousness recovered, and he could breathe spontaneously. Due to the presence of venous thrombosis in the lower extremities, the vascular surgery performed inferior vena cava filter implantation. After a comprehensive evaluation by the department of anesthesiology, on the 25th day after the injury, the departments of plastic surgery and ophthalmology performed open reduction and rigid internal fixation of facial bone fractures and repair of orbital wall fractures under general anesthesia. The patient's occlusal relationship recovered well after the operation, the incision tube was removed, the cannula was cut, the nasal feeding tube was removed, and the patient was able to move freely out of bed and was discharged smoothly. (Fig 1) 

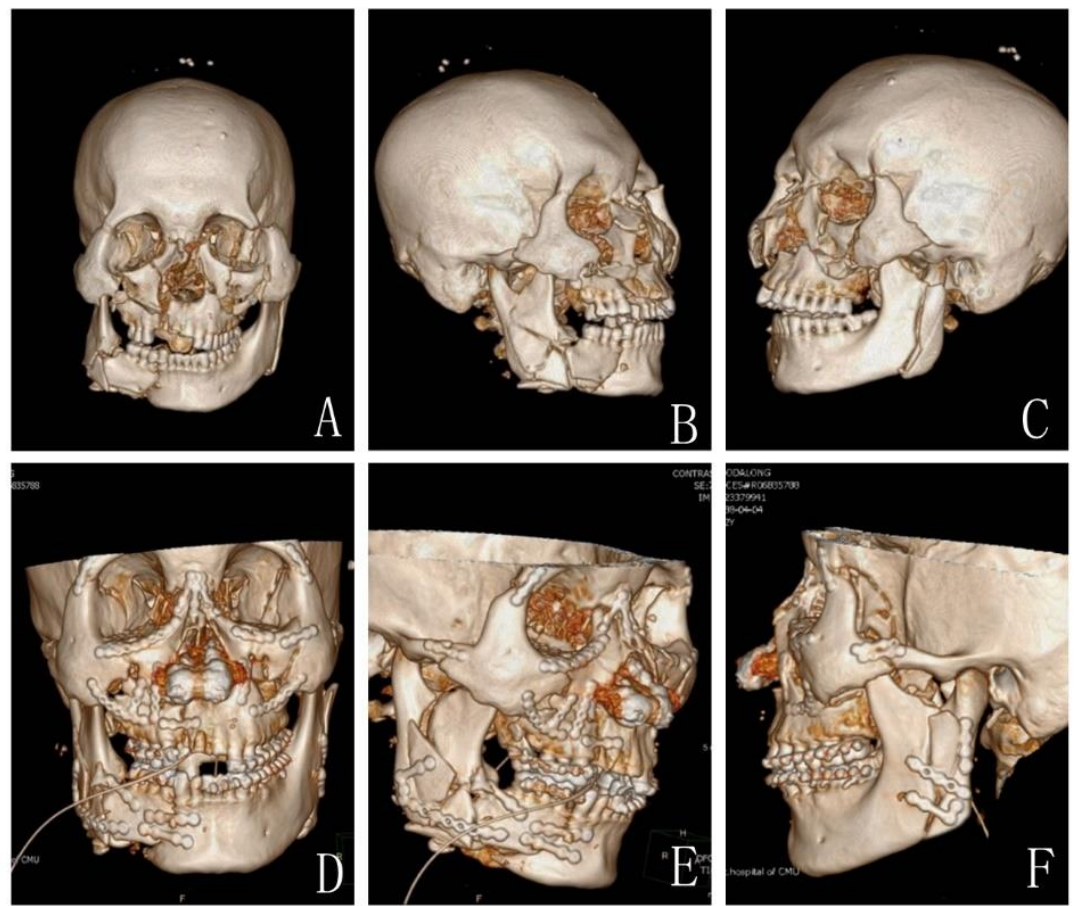

Fig 1 Case 1: ABC Preoperative images of the patient: Before surgery, the patient had multiple fractures of mandible body and ascending ramus, condylar fracture, naso-orbito-ethmoid (NOE) fracture, bilateral zygomaticmaxillary complex fracture, inferior orbital wall fracture, and occlusal disorder. DEF Postoperative photos of patient: The broken end of the fracture reached the standard of anatomical reduction. The occlusal relationship was good and the volume of the orbital cavities was symmetrical.

Case 2 A 20-year-old female patient, 18 weeks of gestation, caused multiple maxillofacial bone fractures and femoral shaft fractures due to a car accident on the routine obstetric check-up. After debridement and suture at the local hospital, she was transferred to the emergency department of our hospital. After consulted by related departments such as plastic surgery, orthopedics, ophthalmology, obstetrics, and anesthesiology, in order to shorten the treatment cycle, plastic surgery, orthopedics, and ophthalmology were combined to complete open reduction and rigid internal fixation of facial bone fractures, orbital wall fracture reconstruction, and open reduction of the right femoral shaft fracture. After the patient's condition was stabilized, the obstetrician gave an induction of labor with the consent of the patient and family members. After the operation, the patient's occlusal relationship recovered well, the facial appearance 
was good, and she was discharged smoothly. (Fig 2)
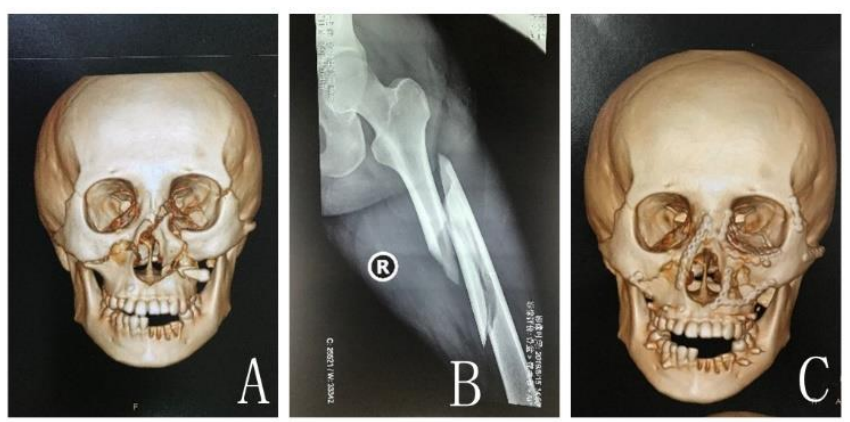

Fig 2 Case 2: A The patient had a type I Lefort fracture, a naso-orbito-ethmoid fracture, and a left zygomaticomaxillary complex fracture preoperatively; B The patient had multiple fractures of the right femoral shaft; $\mathbf{C}$ Anatomical reduction of fracture line was observed after the operation. The patient's facial appearance was good and the occlusal relationship was restored.

\section{Discussion}

The development of specialization and professionalization in modern hospitals has brought many problems to the treatment of multiple injuries. Severe trauma represented by multiple injuries often involves multiple systems and multiple sites, requiring multiple surgical specialists to participate in injury assessment, emergency surgery, and subsequent planned staging operations[3]. At present, the development of hospital disciplines has moved towards specialization and professionalization[4]. Any traditional department cannot cope with the needs of severe trauma treatment, and any single department no longer has the ability to treat severe multiple injuries. In addition, our country's comprehensive 3A hospitals mostly treat patients with chronic diseases, and most patients with acute trauma are not taken seriously. Most beds are reserved for routine patients, and emergency patients are often "difficult to find a bed". According to the current hospital bed management mode and bed turnover rate restrictions, patients need to make an appointment for admission, and beds cannot be reserved for emergency patients, and the mode of ICU admission often needs to correspond to a certain specialty[5]. Joint multidisciplinary trauma treatment should have facilities and capabilities for comprehensive treatment of serious trauma, and the in-hospital trauma treatment system supported by pre-hospital trauma rescue, emergency, trauma surgery, 
surgical anesthesia, ICU, imaging medicine, laboratory medicine, blood transfusion and other trauma medicine core team disciplines. Its basic characteristics are "substantiated multidisciplinary collaborative diagnosis and treatment" and "centralized ward treatment of patients with severe trauma such as multiple injuries". It solves multiple specialized problems at the same time, and is not limited by the performance of conventional beds. This greatly improves the level of treatment for patients with severe trauma[6].

Severe maxillofacial injury is an urgent and critical disease in plastic surgery. This kind of disease comes with dangerous and complicated types of trauma. For the treatment of patients with severe maxillofacial injuries, we should not only pay attention to the injuries of our specialty, and ignore the injuries of other parts and organs of the whole body. Only by timely and effective treatment of serious systemic lifethreatening complications, and close cooperation with multiple disciplines, can we improve the success rate of treatment of this type of disease[7].

The bones of cerebral cranium and facial cranium are closely connected in anatomical structure. Under the action of large external force, the probability of fractures at the same time increases[8]. It can not only cause combined damage to the brain and audiovisual organs, but also easily lead to severe complications such as respiratory obstruction and hemorrhagic shock. Most patients with craniocerebral trauma have disturbances in consciousness, and intracranial hypertension develops rapidly[9]. If decompression is not performed in time, it can cause respiratory failure and death due to brain herniation. For patients with craniocerebral injury and high intracranial pressure, they should be consulted by a neurosurgeon in time, and craniotomy and decompression should be performed if necessary[10]. For patients with respiratory obstruction, such as blood clots or secretions blocked in the mouth and throat, the blood clots or secretions should be removed as soon as possible; if the tongue falls due to a comminuted fracture of the mandible, the tongue should be quickly retracted out of the mouth; when the difficulty of breathing caused by hematoma of the floor of the mouth and swelling of the throat is hard to relieve for a while, to save lives, an emergency tracheotomy should be performed immediately to relieve the respiratory 
obstruction. Case 2 in this group underwent emergency tracheotomy, and the patient's dyspnea was relieved. Because of the rich blood vessels in the maxillofacial region and the large blood vessels, the fierce bleeding after injury can easily lead to hemorrhagic shock[11]. At this time, the bleeding point should be identified quickly and clamped or sutured to stop the bleeding. If the clamp cannot stop the bleeding, pack the sterile gauze or iodoform gauze in the bleeding wound and then pressurized and bandaged to stop the bleeding. At the same time, quickly supplement blood volume via multichannels, preferably whole blood transfusion; give reasonable and adequate fluid replacement, balanced salt solution or lactated Ringer's solution should be preferred; if blood pressure is low, vasoconstrictors can be given appropriately to maintain systolic blood pressure above $8 \mathrm{kPa}[12]$. Patients with rib fractures, limbs and pelvic fractures should be consulted by relevant specialists in thoracic surgery and orthopedics, and those who need to be transferred for treatment should be immediately transferred for treatment. The maxillofacial area should only be subjected to preliminary debridement and suture, and jaw fractures will be treated after being transferred back again[13].

In this group of 173 patients with severe maxillofacial injuries, the close collaboration of anesthesiology, neurosurgery, orthopedics, thoracic surgery and ophthalmology departments has greatly improved the success rate of treatment. The definitive treatment of maxillofacial injuries is mainly the treatment of jaw fractures. For maxillofacial fractures, surgical reduction should be performed immediately after the patient's vital signs are stable and the local swelling subsides, and rigid internal fixation techniques should be used for interosseous fixation[14]. Our experience is that open reduction and rigid internal fixation of jaw fractures is best performed 7-14 days after injury. At this time, the patient's vital signs are stable, local swelling subsides, fractures are easily reset, and the trauma is small. If the patient's condition is complicated and cannot tolerate the operation, the timing of the operation can be appropriately extended to about 6 weeks after the injury.

\section{Conclusion}

At present, due to insufficient professional cooperation, poor management coordination, and inconvenient transportation and communication, the effect of early treatment of 
craniofacial injuries in our country is still unsatisfactory, and there is still a certain gap between China and western developed countries. Multidisciplinary treatment of craniomaxillofacial trauma overcomes the limitations of traditional single discipline and single treatment system, and can provide individualized comprehensive treatment plans for specific injuries. It not only helps improve the success rate of first aid, but also reduces post-injury deformities and dysfunctions.

\section{Acknowledgements}

Not applicable.

\section{Funding}

This work was supported by the Key Special Project of Ministry of Science and Technology(2019YFF0302403), the China Postdoctoral Science Foundation (2018M641741) and the Doctoral Research Initiation Fund of Liaoning Province(2019BS-281). The funding had no role in study design, collection, analysis or interpretation of data, or manuscript preparation.

\section{Availability of data and materials}

We declared that materials described in the manuscript, including all relevant raw data, will be freely available to any scientist wishing to use them for non-commercial purposes. The datasets used and analyzed during the current study are available from the corresponding author upon reasonable request without breaching participant confdentiality.

\section{Declarations}

\section{Ethics approval and consent to participate}

This study has been approved by the Ethics Committee of The First Hospital of China Medical University. Informed consent is waived for this retrospective study by the Ethics Committee of The First Hospital of China Medical University. We confirmed all methods were carried out in accordance with relevant guidelines and regulations.

\section{Consent for publication}

All authors agree to publish. 


\section{Competing interests}

All authors have no conflict of interest.

\section{Author details}

Department of Plastic Surgery, The First Hospital of China Medical University, No.155,Nanjing North Street, Shenyang 110001, China.

\section{References}

1. Tekin A, Bahși I. Global Research on Maxillofacial Fracture Over the Last 40 Years: A Bibliometric Study. The Journal of craniofacial surgery. 2021. doi: 10.1097/SCS.0000000000007627.

2. Daniels J, Albakry I, Braimah R, Samara M, Albalasi R, Begum F, Al-Kalib M. Experience with Airway Management and Sequencing of Repair of Panfacial Fractures: A Single Tertiary Healthcare Appraisal in Najran, Kingdom of Saudi Arabia-A Retrospective Study. Annals of maxillofacial surgery. 2020;10(2):402-408.

3. Alves L, Aragão I, Sousa M, Gomes E. Pattern of maxillofacial fractures in severe multiple trauma patients: a 7-year prospective study. Brazilian dental journal.2014; 25(6):561-564.

4. Nishimoto R, Dodson T, Lang M. Is the Mandible Injury Severity Score a Valid Measure of Mandibular Injury Severity? Journal of oral and maxillofacial surgery : official journal of the American Association of Oral and Maxillofacial Surgeons.2019;77(5):1023-1030.

5. Wei S, Li B, Bai Y, Li C, Zhang X. Multidisciplinary integrated treatment for cranio-maxillofacial trauma. Zhonghua kou qiang yi xue za zhi.2015; 50(4):214-216.

6. Long A, Lefebvre C, Masneri D, Mowery N, Chang M, Johnson J, Carter J. The Golden Opportunity: Multidisciplinary Simulation Training Improves Trauma Team Efficiency. Journal of surgical education.2019;76(4):1116-1121.

7. Zhong X, Wen X, Ji C, Fei X, Zhao X. A temporary-sustainable team: A new multidisciplinary team model for severe trauma. Zhonghua chuang shang za zhi.2020; 23(6):363-366.

8. Pappachan B, Alexander M. Biomechanics of cranio-maxillofacial trauma. J Maxillofac Oral Surg.2012; 11(2):224-230.

9. O'Donnell J, Browne K, Kilbaugh T, Chen H, Whyte J, Cullen D. Challenges and demand for modeling disorders of consciousness following traumatic brain injury. Neuroscience and biobehavioral reviews. 2019; 98:336-346.

10. Amin D, Cooper M, Newton K. Traumatic Lingual Hematoma Resulting in Bilateral Temporal Mandibular Joint Dislocations. The Journal of emergency medicine. 2018; 54(5):e101-e103.

11. Louro J, Varon AJ. Airway management in trauma. International anesthesiology 
clinics. 2021;59(2):10-16.

12. Jose A, Nagori S, Agarwal B, Bhutia O, Roychoudhury A. Management of maxillofacial trauma in emergency: An update of challenges and controversies. Journal of emergencies, trauma, and shock. 2016; 9(2):73-80.

13. Das D, Salazar L, Zaurova M. Maxillofacial trauma: managing potentially dangerous and disfiguring complex injuries. Emergency medicine practice. 2017; 19:S1-S2.

14. Strong EB, Gary C. Management of Zygomaticomaxillary Complex Fractures. Facial Plast Surg Clin North Am. 2017; 25(4):547-562. 

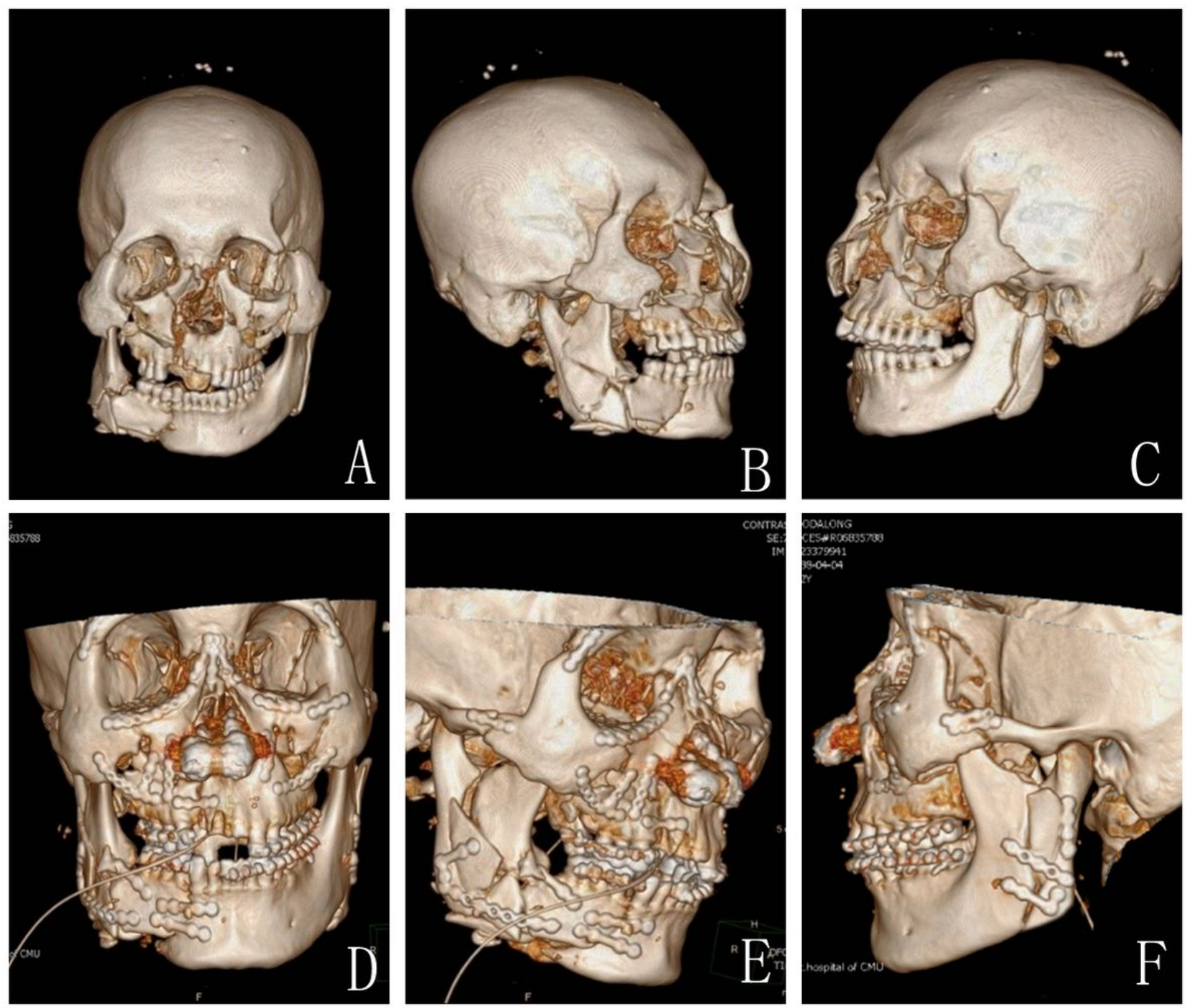

Figure 1

Case 1: ABC Preoperative images of the patient: Before surgery, the patient had multiple fractures of mandible body and ascending ramus, condylar fracture, naso-orbito-ethmoid $₫$ NOE $\triangle$ fracture, bilateral zygomaticmaxillary complex fracture, inferior orbital wall fracture, and occlusal disorder. DEF Postoperative photos of patient $\bigotimes T$ he broken end of the fracture reached the standard of anatomical reduction. The occlusal relationship was good and the volume of the orbital cavities was symmetrical. 

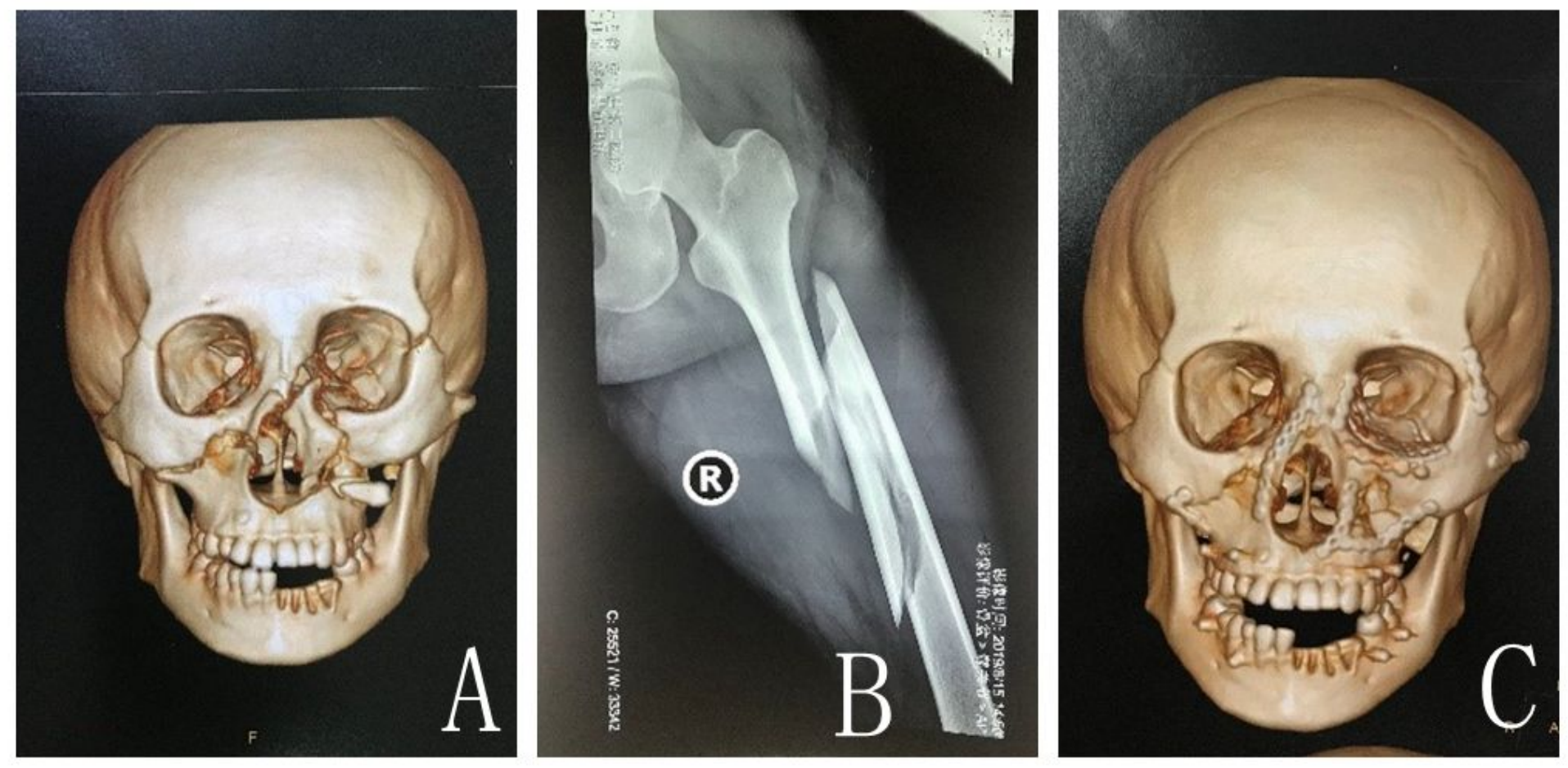

\section{Figure 2}

Case 2: A The patient had a type I Lefort fracture, a naso-orbito-ethmoid fracture, and a left zygomaticomaxillary complex fracture preoperatively; B The patient had multiple fractures of the right femoral shaft; $C$ Anatomical reduction of fracture line was observed after the operation. The patient's facial appearance was good and the occlusal relationship was restored. 